\title{
CUCURBITACINAS E SUAS PRINCIPAIS CARACTERÍSTICAS ESTRUTURAIS
}

\section{Ligia Maria Marino Valente*}

Departamento de Química Orgânica, Instituto de Química, Universidade Federal do Rio de Janeiro, Centro de Tecnologia, Bloco A, Ilha do Fundão, 21910-240 Rio de Janeiro - RJ

Recebido em 16/7/03; aceito em 9/3/04; publicado na web em 27/8/04

CUCURBITACINS AND THEIR MAIN STRUCTURAL CHARACTERISTICS. The cucurbitacins are highly oxygenated triterpenoid compounds found in several botanical families that show high toxicity and varied biological activities. This review presents the main cucurbitacins so far isolated and their key structural characteristics. It complements and updates the existing reviews on this subject.

Keywords: cucurbitacin; triterpenoid; review.

\section{INTRODUÇÃO}

Cucurbitacinas são, por definição, triterpenos altamente oxigenados, com o esqueleto biogeneticamente incomum $19(10 \rightarrow 9 \beta)$ abeo-10 $\alpha$-lanostano (cucurbitano) (Figura 1), que podem ser encontrados livres ou glicosilados ${ }^{1}$.

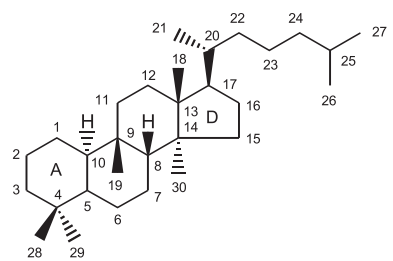

Figura 1. Esqueleto cucurbitano

As primeiras agliconas isoladas com esqueleto cucurbitano receberam como nome a palavra cucurbitacina seguida de uma letra, de acordo com a cronologia do isolamento/elucidação estrutural (Figura 2). No entanto isso não se constituiu numa regra geral, encontrando-se atualmente diversos nomes para essas substâncias.

Elas são reconhecidas principalmente como os princípios tóxi$\cos ^{1}$ das plantas da família Cucurbitaceae, estando presentes em algumas espécies dessa família botânica usadas na medicina popular brasileira como a "buchinha" (Luffa operculata) 2,3, "taiuiá" (Wilbrandia ebracteata ${ }^{4-6}$ e/ou Cayaponia tayuya ${ }^{7,8}$ ) e "nhandiroba"

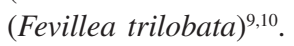

Embora encontradas predominantemente em espécies da família Cucurbitaceae, estão presentes também em várias outras famílias botânicas $^{11,12}$, como Begoniaceae, Chrysobalanaceae ${ }^{13}$, Cruciferae, Datiscaceae, Desfontaniaceae, Elaeocarpaceae, Flacourtiaceae ${ }^{14}$, Lauraceae, Liliaceae, Polemoniaceae, Primulaceae, Rosaceae ${ }^{15}$, Rubiaceae, Saxifragaceae ${ }^{16}$, Scrophulariaceae, Sterculiaceae e Thymelaeaceae ${ }^{17}$, e também na espécie de cogumelo venenoso Hebeloma vinosophyllum ${ }^{18}$.

O grande interesse que essas substâncias têm despertado está relacionado principalmente à sua toxicidade e ao seu amplo espectro de atividades biológicas. Esse assunto foi revisto por Miró ${ }^{11}$ que destacou as atividades citotóxica, antitumoral ${ }^{19}$, antiinflamatória,

*e-mail: valente@iq.ufrj.br

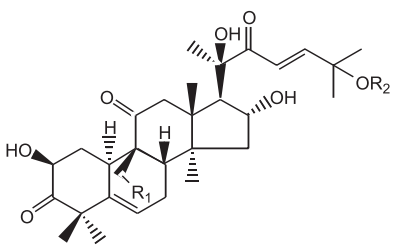

Cucurbitacina $A \quad \mathrm{R}_{1}=\mathrm{OH}, \mathrm{R}_{2}=\mathrm{Ac}$ Cucurbitacina $B \quad R_{1}=H, R_{2}=A c$ Cucurbitacina D $\mathrm{R}_{1}=\mathrm{R}_{2}=\mathrm{H}[31]$

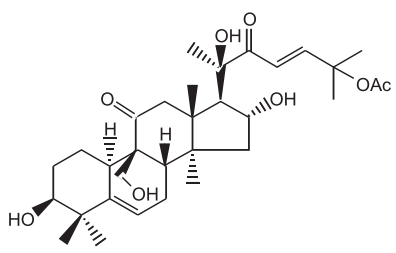

Cucurbitacina C

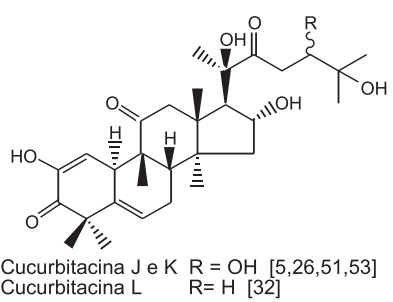

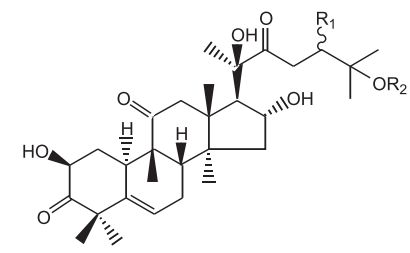

Cucurbitacina $\mathrm{G}$ e H $\quad \mathrm{R}_{1}=\mathrm{OH}, \mathrm{R}_{2}=\mathrm{H}[5]$ $\begin{array}{ll}\text { Diidrocucurbitacina } B & \mathrm{R}_{1}=\mathrm{H}, \mathrm{R}_{2}=A c \\ \text { Cucurbitacina } \mathrm{R} & \mathrm{R}_{1}=\mathrm{R}_{2}=\mathrm{H}[5]\end{array}$

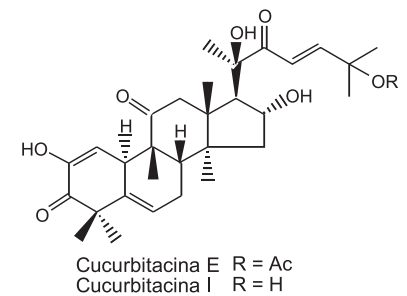
Cucurbitacin

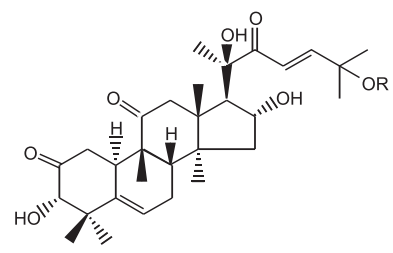

Isocucurbitacina $\mathrm{B} \quad \mathrm{R}=\mathrm{Ac}$ $\begin{array}{ll}\text { Isocucurbitacina } B & R=A c \\ \text { Isocucurbitacina } D & R=H \quad[62]\end{array}$

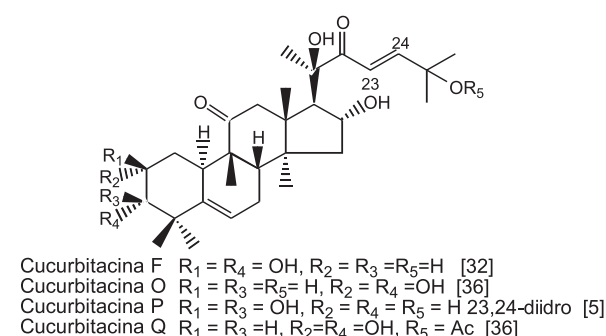

Figura 2. Estruturas das cucurbitacinas mais conhecidas (ver refs. 1, 11 e as citadas entre colchetes)

antifertilizante, fago-repelente, hepato protetora e curativa e antimicrobiana como as mais significativas. Recentemente as cucurbitacinas foram utilizadas como marcador biogenético na quimiossistemática da superordem Violiflorae ${ }^{20}$. 
Estruturalmente são classificadas de acordo com suas funcionalidades no anel A, modificações na cadeia lateral e considerações estereoquímicas. O padrão de oxigenação inclui um grupamento hidroxila ou carbonila no carbono $3^{21}$, além da possibilidade de oxigenação nos carbonos 2, 7, 11, 16, 19, 20, 22 e 25.

A história do isolamento e determinação estrutural das cucurbitacinas confunde-se com a própria história da fitoquímica. A primeira substância isolada, a elaterina ${ }^{22}$ (mais tarde denominada cucurbitacina E, Figura 2), data de 1831 e, como todas as substâncias naturais isoladas de plantas medicinais no século XIX, só teve sua estrutura elucidada muitos anos mais tarde, em função do desenvolvimento da teoria estrutural e das técnicas experimentais.

Até o momento várias cucurbitacinas foram isoladas, incluindo algumas com modificações estruturais como as nor $^{5,8-10,23-25}$, hexanor ${ }^{26-30}$, heptanor ${ }^{9}$ octanor $^{26}$ e neocucurbitacinas ${ }^{3}$ (Figuras 2, 3 e 4).
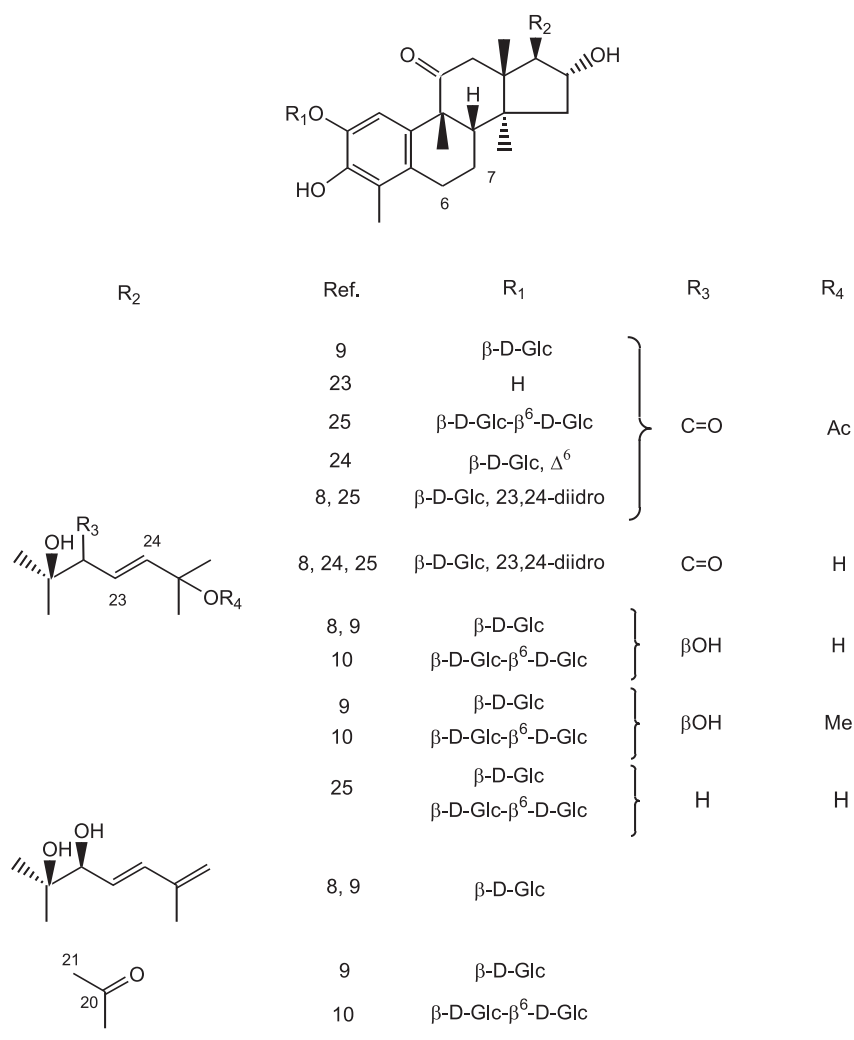

Figura 3. Exemplos de estruturas de nor e heptanorcucurbitacinas

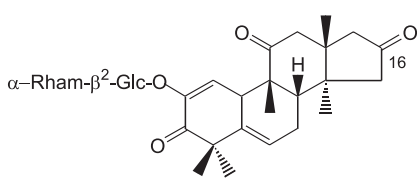

octanorcucurbitacina [26]

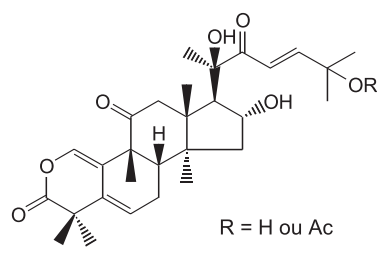

neocucurbitacina [3]
Figura 4. Estruturas das octanor e neocucurbitacina isoladas até o momento (referências entre colchetes)

Neste trabalho estão compiladas as principais cucurbitacinas isoladas até o momento, agrupadas de acordo com suas características estruturais. $\mathrm{O}$ trabalho tem como objetivo complementar e atualizar as duas principais revisões ${ }^{1,11}$ publicadas até o momento sobre essas substâncias, proporcionando uma visão abrangente sobre as estrutu- ras das mesmas e facilitando o acesso aos principais dados relacionados à sua elucidação estrutural.

\section{PRINCIPAIS CARACTERÍSTICAS ESTRUTURAIS}

Todo o histórico da elucidação estrutural das cucurbitacinas até 1970, incluindo a determinação da estereoquímica dos centros quirais de seu esqueleto, foi detalhadamente revisto por Lavie e Glotter ${ }^{1}$. Os trabalhos descritos basearam-se principalmente em transformações químicas, análises de Dispersão Óptica Rotatória (DOR) e Ressonância Magnética Nuclear de ${ }^{1} \mathrm{H}\left(\mathrm{RMN}{ }^{1} \mathrm{H}\right)$ de vários derivados em comparação com modelos. Com a evolução das técnicas de separação e elucidação estrutural, várias estruturas foram sendo isoladas. Velde ${ }^{31}$ compilou e comparou os deslocamentos químicos em RMN ${ }^{13} \mathrm{C} \mathrm{e}{ }^{1} \mathrm{H}$ de nove diferentes cucurbitacinas, mostrando o assinalamento inequívoco dos átomos de carbono dessas substâncias. $\mathrm{Che}^{32} \mathrm{e}$ Sasamori $^{33}$ realizaram trabalho semelhante em $\mathrm{RMN}{ }^{1} \mathrm{H}$. Budzikiewiez $^{34}$, Audier ${ }^{35}$ e Kupcham ${ }^{36}$ analisaram o espectro de massas de algumas cucurbitacinas propondo fragmentos típicos para essa classe de substâncias. Esses dados foram confirmados e ampliados principalmente nos trabalhos de Johnson ${ }^{37,38}$ pela utilização de Espectrometria de Massas de Alta Resolução (EMAR) e variação nas formas de ionização. Hoje com as modernas técnicas de RMN em duas dimensões, Difração de Raio $\mathrm{X}^{39,40}$ e técnicas hifenadas ${ }^{41}$, está sendo possível ampliar cada vez mais o universo estrutural das cucurbitacinas, detectando estruturas mais complexas mesmo minoritárias.

O conhecimento acumulado até o momento tem mostrado que as principais características estruturais de diferenciação das cucurbitacinas estão localizadas no anel A e na cadeia lateral. Um dado adicional, levado em conta neste artigo, é a análise dos grupamentos carbonila (localização e características), presentes de maneira sistemática na grande maioria dessas substâncias.

\section{Anel A}

São encontradas até o momento doze variações estruturais de

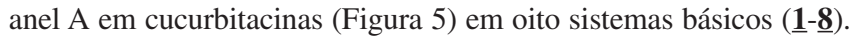
Dentre esses, os sistemas $\underline{\mathbf{1}}$ e $\underline{\mathbf{2}}$ foram os primeiros a serem descritos $^{1}$. Foi também (em 1960) através da observação de que o proton olefínico (H-1) do sistema diosfenólico (grupamento $\alpha$-dicetona enolizado) presente em 1 apresentava em $\mathrm{RMN}{ }^{1} \mathrm{H}$ um sinal duplo em $\delta 5,97(J=\sim 3 \mathrm{~Hz})$, que se confirmou a presença de um hidrogênio em $\mathrm{C}$-10, surgindo assim uma das primeiras evidências do esqueleto lanostano rearranjado das cucurbitacinas ${ }^{1}$.

São denominadas isocucurbitacinas ${ }^{2,30,59,62}$ aquelas que possuem o sistema $\underline{3 \mathbf{a}}\left(3 \alpha \mathrm{OH}\right.$, equatorial) e epi-isocucurbitacinas ${ }^{59,62}$ as de sistema $\underline{3 \mathbf{b}}(3 \beta \mathrm{OH}$, axial).

Cucurbitacinas contendo no anel A apenas uma hidroxila em C-3, $\underline{\mathbf{5}} \mathbf{a}$ e $\underline{\mathbf{5 b}}$, são geralmente encontradas na natureza como glicosídeos e podem apresentar um esqueleto menos oxigenado que as outras cucurbitacinas. A orientação da hidroxila em C-3 é de um

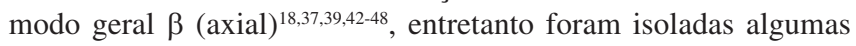
cucurbitacinas glicosiladas com o substituinte em C-3 (OGli) $\alpha$ orien$\operatorname{tado}^{49,50}$

No sistema $\underline{\mathbf{4}}$, 2,3-di-hidroxi, as variações $\underline{\mathbf{4 b}}[2 \beta, 3 \alpha$ (diequatorial) $]^{15,28,29,32,64}$ e $\underline{\mathbf{4 c}}[2 \beta \text { (equatorial), } 3 \beta \text { (axial) }]^{4,5,27,29,30,52,55,58,63}$ são as de maior ocorrência. A determinação da estereoquímica relativa desses centros é geralmente assinalada por RMN, principalmente através da observação da constante de acoplamento de H-3 $3^{4,27,64}$.

São denominadas norcucurbitacinas as que possuem o sistema 6. Nelas o anel A está aromatizado, com perda de um dos grupos metilas geminais ligados a C-4. São em sua grande maioria glico- 
siladas em C-2 e foram isoladas de espécies latino-americanas da família Cucurbitaceae $e^{5,8-10,23-25}$ (ver também Figura 3).

Cucurbitacinas com o anel A rearranjado com perda de um átomo de carbono e formação de um anel $\delta$-lactona C-1/C-3 foram isoladas recentemente e denominadas neocucurbitacinas ${ }^{3}, \underline{7}$ (ver também Figura 4).
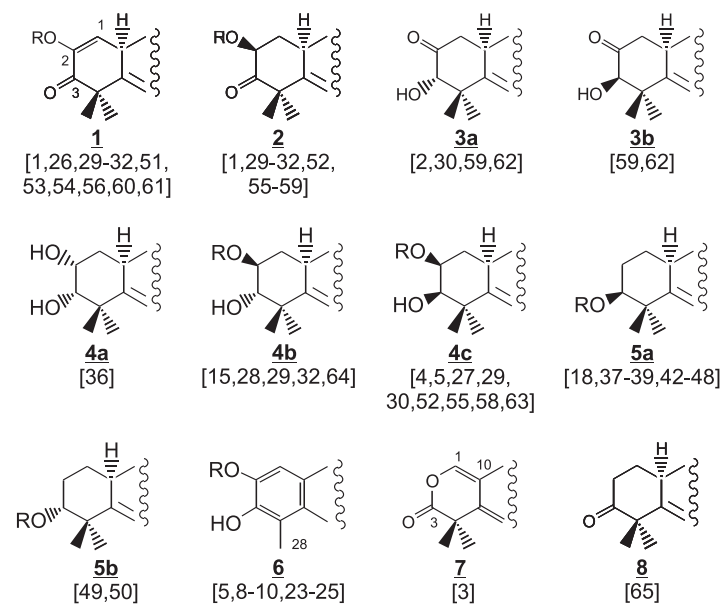
$30,52,55,58,63$
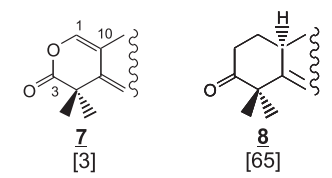

$\mathrm{R}=\mathrm{H}$ ou Glicosídeo

Figura 5 - Principais tipos de anel A encontrados em cucurbitacinas (referências entre colchetes)

\section{Cadeia lateral}

A constatação de que as cucurbitacinas possuíam uma cadeia lateral com oito átomos de carbono foi um dos fatores que contribuíram para a elucidação do esqueleto dessas substâncias. Os trabalhos pioneiros de identificação estrutural utilizaram basicamente transformações químicas que incluíam várias reações de clivagem ${ }^{1}$. As primeiras a serem identificadas e de ocorrência majoritária até hoje, foram as acíclicas com um grupamento carbonila em C-22 e com ou sem insaturação em C-23,24 (ㅁ, Figura 6; Cucurbitacinas A-F, L, OR, Figura 2).

Atualmente podemos encontrar vários tipos de cadeia lateral em cucurbitacinas (Figura 6) variando desde as acíclicas não-oxigenadas $^{18,47}, \underline{\mathbf{1 1}}$, até tetra-oxigenadas ${ }^{1,26,39,42,51}, \underline{\mathbf{1 0}}$ e $\underline{\mathbf{1 7}}$, além das cíclicas, que resultam de reações intramoleculares entre diferentes átomos de carbono (C-23, C-24 ou C-25) e átomos de oxigênio, presentes na cadeia lateral $(\mathrm{C}-20)^{37,38,50-52}, \underline{\mathbf{2 3}}, \underline{\mathbf{2 5}}$, e $\underline{\mathbf{2 6}}$, ou no anel D $(\mathrm{C}-16)^{4,26,30,53,54}$, $\underline{22}$ e $\underline{24}$

A determinação da estereoquímica dos centros quirais presentes nas cadeias laterais acíclicas (substituinte hidroxila em C-22, 23 e 24, Figura 6) tem se mostrado um desafio. Okabe ${ }^{39}$ e Achenbach ${ }^{49}$ através de transformações químicas, análise detalhada por $\mathrm{RMN}{ }^{1} \mathrm{H}$ e ${ }^{13} \mathrm{C}$ (incluindo comparação com modelos) e Difração de Raio $\mathrm{X}^{39}$ conseguiram determinar a estereoquímica desses centros nos sistemas estudados ( $\underline{\mathbf{1 0}}$ e $\underline{\mathbf{1 8}}$ ). Outros autores ${ }^{9,26}$ utilizaram comparação de dados espectroscópicos de estruturas modelo nessa determinação, $\underline{13}$ e $\underline{18}$. Nos sistemas $\underline{12}$ e $\underline{17}$ (24-hidroxi) a estereoquímica do centro C-24 permanece não-determinada. Oobayashi ${ }^{44}$ propôs uma configuração 24- $R$ para 12, sem no entanto apresentar evidências conclusivas para tal. A mesma incerteza quanto a estereoquímica é encontrada nos tipos de cadeia lateral $\underline{15}$ e $\underline{16}$.

Algumas cucurbitacinas sofreram perda parcial da cadeia lateral, como as hexanor ${ }^{26-30}$ e heptanorcucurbitacinas ${ }^{9}$ (manutenção de C-20 e C-21), $\underline{\mathbf{2 0}}$, (ver também Figuras 3 e 7) e recentemente foi isolada uma cucurbitacina ${ }^{26}$ sem a cadeia lateral (octanorcucurbitacina, Figura 4).

\section{Carbonilas}

Embora existam relatos de cucurbitacinas sem grupamento carbonila $18,39,40,42,45,46,48$, ele está presente de maneira sistemática na grande maioria dessas substâncias. São de um modo geral carbonilas cetônicas, conjugadas ou não a ligações duplas (Figura 7), e em raros casos aldeídicas ${ }^{46-48}, \underline{\mathbf{3 8}}$, ou de grupo carboxílico de ester ${ }^{3}, \underline{\mathbf{3 0}}$.

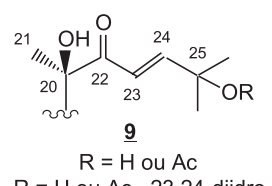

$R=H$ ou Ac, $23,24-$ diidro [refs: Fig. 7]<smiles>[R]C(C)(C)[C@H](O)CCC(C)C</smiles>

$\mathrm{R}=\mathrm{H}$ ou Gli [43-45]<smiles>CC(C)C(=O)C[C@@H](O)C(C)(C)O</smiles><smiles>[AsH3-][PbH3+2]</smiles><smiles>C=C(C)CCC(=O)C(C)(C)O</smiles>

$\frac{19}{[25]}$<smiles>CC1(C)CCC(=O)[C@@]2(C)CC[C@@]1(C)O2</smiles>

23

$[37,38,51]$<smiles>CC(C)[C@@H](O)[C@@H](O)[C@@H](O)C(C)(C)O</smiles><smiles>[R]C(C)=CCCC(C)C</smiles>
$\mathrm{R}_{1}=\mathrm{R}_{2}=\mathrm{CH}_{3}[18,47]$ $\mathrm{R}_{1}=\mathrm{R}_{2}=\mathrm{CH}_{3}$ 24,25-diidro [47] [45] $\left\{\begin{array}{l}\mathrm{R}_{1}=\mathrm{CH}_{3}, \mathrm{R}_{2}=\mathrm{CH}_{2} \mathrm{OH} \text { ou } \mathrm{CH}_{2} \mathrm{OGli} \\ \mathrm{R}_{1}=\mathrm{CH}_{2} \mathrm{OH} \text { ou } \mathrm{CH}_{2} \mathrm{OGli}, \mathrm{R}_{2}=\mathrm{CH}_{3}\end{array}\right.$ $[39,42]$<smiles>C=C(C)/C=C/[C@@H](O)C(C)(C)O</smiles><smiles>[R2]C(C)=CCC([R1])C(C)(C)O</smiles>

$\mathrm{R}_{1}=\mathrm{OH}, \mathrm{R}_{2}=\mathrm{H}[55]$ $\begin{array}{cl}\underline{13} & \mathrm{R}_{1}==\mathrm{O}, \mathrm{R}_{2}=\mathrm{H}[27] \\ {[8,9]} & \mathrm{R}_{1}=\mathrm{H}, \mathrm{R}_{2}=\mathrm{CH}_{2} \mathrm{OH}[52]\end{array}$<smiles>[R]C([C@H](O)C=C(C)C)C(C)(C)C</smiles>
15 $\mathrm{R}=\mathrm{OH}[42]$ $\mathrm{R}=\mathrm{H}[46,48]$

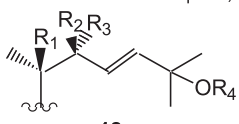
18 $\mathrm{R}_{1}=\mathrm{R}_{3}=\mathrm{OH}, \mathrm{R}_{2}=\mathrm{R}_{4}=\mathrm{H}[26]$ $R_{1}=R_{2}=O H, R_{3}=H, R_{4}=H$ ou CH $3[8-10,25,26,49]$ $R_{1}=R_{2}=R_{3}=H, R_{4}=H$ ou $\mathrm{CH}_{3}[59,48]$ $\mathrm{R}_{1}=\mathrm{OH}, \mathrm{R}_{2}=\mathrm{R}_{3}=\mathrm{H}, \mathrm{R}_{4}=\mathrm{H}$ ou Gli [25]

$[9,26-30]$<smiles>CCCC(C)(C)OC(=O)/C=C\C(=O)O</smiles>

[27]<smiles>CC(C)C[C@H](O)[C@@H](O)C(C)(C)O</smiles>

[42]

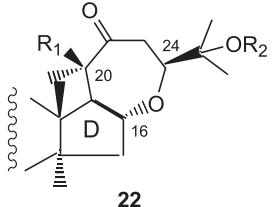

$\mathrm{R}_{1}=\mathrm{R}_{2}=\mathrm{H}[54]$ $\mathrm{R}_{1}=\mathrm{OH}, \mathrm{R}_{2}=\mathrm{CH}_{3}[53$

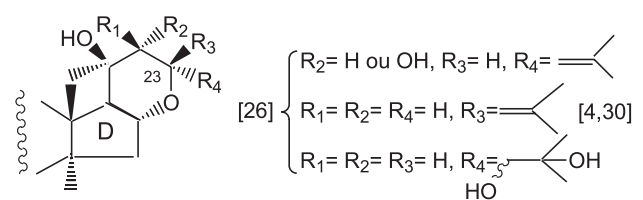

$\underline{24}$

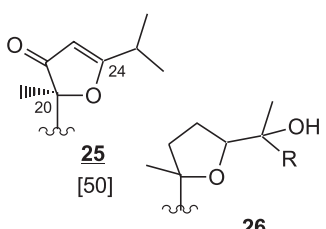

$\mathrm{R}=\mathrm{CH}_{3}$ ou $\mathrm{CH}_{2} \mathrm{OH}[52]$

Figura 6. Principais tipos de cadeia lateral encontrados em cucurbitacinas (referências entre colchetes) 
Estão localizados com maior freqüência nos carbonos 2, 3, 11 e 22. No caso de hexanor ${ }^{26-30}$ e heptanor ${ }^{9}$ cucurbitacinas, em que há perda da cadeia lateral, aparece um grupamento carbonila no carbono $20, \underline{\mathbf{3 3}}$, e em octanorcucurbitacinas no carbono $16^{26}, \underline{\mathbf{3 4}}$. Foi isolada uma cucurbitacina carbonilada no carbono $7^{47}, \underline{37}$, e duas no carbono $19^{46,48}, \underline{\mathbf{3 8}}$
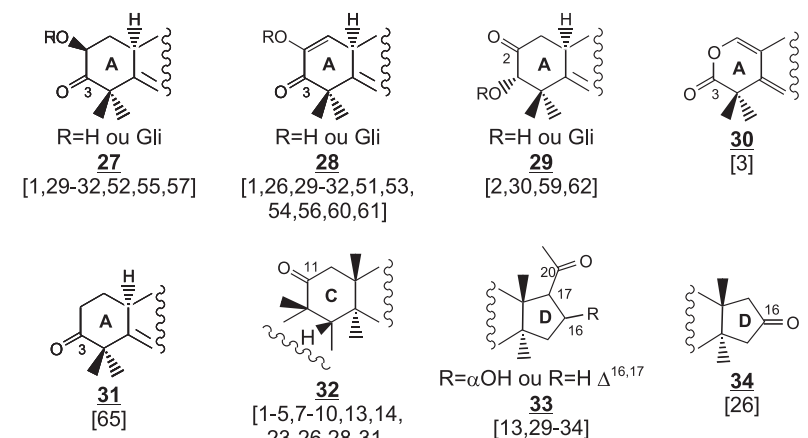

$[1-5,7-10,13,14$,

23-26, 28-31,

$36,38,43,44$,

49-59,61-64]

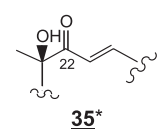

$\left[1,5,8,9, \frac{35}{13}, 15,17,23\right.$

$25,27,29,30,52$

$55-59,61-63]$

$$
\begin{aligned}
& \underbrace{\mathrm{O}}_{22} \\
& 3^{*} \text { * } \\
& {[1,5,7,8,15} \\
& \text { 24-27,44,59] }
\end{aligned}
$$
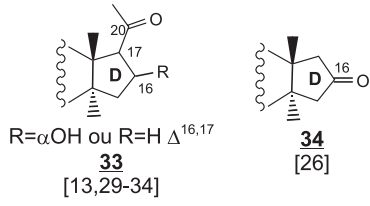

*A localização da carbonila em C-22 pode ser encontrada também em outros tipos menos comuns de cadeia lateral. Ver Figura 6 como complemento.

Figura 7. Principais localizações e características dos grupamentos carbonila encontrados em cucurbitacinas (referências entre colchetes)

\section{CONCLUSÃO}

A elucidação estrutural das cucurbitacinas ocupa, sem dúvida, um lugar importante na história da química de produtos naturais. Os trabalhos pioneiros contaram com o brilhantismo de químicos como Derek H. R. Barton, Otto R. Gottlieb e David Lavie entre muitos outros, em elegantes transformações que levaram à elucidação de várias estruturas, em uma época com poucos recursos em métodos físicos de análise. O desafio ainda permanece, quer no re-estudo de algumas estruturas à luz de técnicas analíticas mais modernas, quer no isolamento e elucidação de novas substâncias, assim como na pesquisa de seu potencial terapêutico.

\section{REFERÊNCIAS}

1. Lavie, D.; Glotter, E.; Fortchr. Chem. Org. Natust. 1971, 29, 307.

2. Matos, F. J.; Gottlieb, O. R.; An. Acad. Bras. Ci. 1967, 39, 245.

3. Kawahara, N.; Kurata, A.; Hakamatsuka, T.; Sekita, S.; Satake, M.; Chem. Pharm. Bull. 2001, 49, 1377.

4. Schenkel, E. P.; Farias, M. R.; Mayer, R.; Breitmaier, E.; Rücker, G.; Phytochemistry 1992, 31, 1329

5. Farias, M. R.; Schenkel, E. P.; Mayer, R.; Rücker, G.; Planta Med. 1993 59, 272.

6. Almeida, F. R. C.; Rao, V. S. N.; Matos, M. E. O.; Phytother. Res. 1992, 6, 189; Peters, R. R.; Farias, M. R.; Ribeiro-do-Valle, R. M.; Planta Med. 1997, 63, 525; Peters, R. R.; Saleh, T. F.; Lora, M.; Patry, C.; Fernandes, A. J. B.; Farias, M. R.; Ribeiro-do-Vale, R. M.; Life Sci. 1999, 64, 2429.

7. Bauer, R.; Berganza, L. H.; Seligmann, O.; Wagner, H.; Phytochemistry $1985,24,1587$

8. Himeno, E.; Nagao, T.; Honda, J.; Okabe, H.; Irino, N.; Nakasumi, T.; Chem. Pharm. Bull. 1992, 40, 2885.

9. Valente, L. M. M.; Gunatilaka, A. A. L.; Glass, T. E.; Kingston, D. G. I.; Pinto, A. C.; J. Nat. Prod. 1993, 56, 1772.

10. Valente, L. M. M.; Gunatilaka, A. A. L.; Kingston, D. G. I.; Pinto, A. C.; J. Nat. Prod. 1994, 57, 1560.

11. Miró, M.; Phytother. Res. 1995, 9, 159

12. Uma listagem correlacionando as principais cucurbitacinas isoladas com 91 espécies de plantas, agrupadas por família botânica, pode ser encontrada na ref. 11. As famílias botânicas não contempladas na citada referência estão correlacionadas no texto às suas respectivas referências bibliográficas.

13. Oberlies, N. H.; Burgess, J. H.; Navarro, H. A.; Pinos, R. E.; Soejarto, D. D.; Farnsworth, N. R.; Kinghorn, A. D.; Wani, M. C.; Wall, M. E.; J. Nat. Prod. 2001, 64, 497.

14. Beutler, J. A.; McCall, K. L.; Herbert, K.; Herald, D. L.; Pettit, G. R.; Johnson, T.; Shoemaker, R. H.; Boyd, M. R.; J. Nat. Prod. 2000, 63, 657.

15. Konoshima, T.; Kashiwada,Y.; Takasaki, M.; Kozuka, M.; Yasuda, I.; Cosentino, L. M.; Lee, K-H.; Bioorg. Med. Chem. Lett. 1994, 4, 1323; Munoz, O.; Delporte, C.; Blackhouse, N.; Erazo, S.; Negret, R.; Maldonado, S.; Lopez-Perez, J. L.; San Feliciano, A.; Z. Naturforsch., C: J. Biosci. 2000, 55, 141.

16. Arisawa, M.; Takeshima, Y.; Bai, H.; Hayashi, T.; Morita, N.; Soyakugaku Zasshi 1993, 47, 334 (CA 120:73484).

17. Fuller, R. W.; Cardellina, J. H.; Gragg, G. M.; Boyd, M. C.; J. Nat. Prod. 1994, 57, 1442.

18. Fujimoto, H.; Hagiwara, H.; Suzuki, K.; Yamazaki, M.; Chem. Pharm. Bull. 1987, 35, 2254.

19. Cassady, J. M.; Sufness, M. Em Anticancer Agents Based on Natural Product Models; Cassady, J. M.; Douros, J. D., eds.; Academic Press: New York, 1980, cap. 7.

20. Paggotto, C. L.; Kaplan, M. A. C.; Gottlieb, O. R.; An. Acad. Bras. Ci. 1995, 67 (Supl. 3), 439.

21. Zander, J. M.; Wigfield, D. C.; Chem. Commun. 1970, 1599.

22. Berg, A.; Bull. Soc. Chim. France 1906, 35, 435.

23. Achenbach, H.; Hefter-Bübl, U.; Constenla, M. A.; J. Chem. Soc., Chem. Commun. 1987, 441

24. Matos, M. E. O.; Machado, M. I. L.; Craveiro, A. A.; Matos, F. J. A.; BrazFilho, R.; Phytochemistry 1991, 30, 1020.

25. Achenbach, H.; Waibel, R.; Hefter-Bübl, U.; Constenla, M. A.; J. Nat. Prod. 1993, 56, 1506.

26. Kanchanapoom, T.; Kasai, R.; Yamasaki, K.; Phytochemistry 2002, 59, 215.

27. Stuppner, H.; Müller, E. P.; Wagner, H.; Phytochemistry 1990, 29, 305.

28. Ohsaki, A.; Kubota, T.; Asaka, Y.; Phytochemistry 1990, 29, 1330.

29. Fang, X.; Phoebe, C. H.; Pezzuto, J. M.; Fong, H. H. S.; Farnsworth, N. R.; Yellin, B.; Hecht, S. M.; J. Nat. Prod. 1984, 47, 988.

30. Rao, M. M.; Meshulam, H.; Lavie, D.; J. Chem. Soc., Perkin Trans.l 1974, 2552.

31. Velde, V. V.; Lavie, D.; Tetrahedron 1983, 39, 317.

32. Che, C-T.; Fang, X.; Phoebe, C. H.; Kinghorn, A. D.; Farsworth, N. R.; Yellin, B.; Hecht, S. M.; J. Nat. Prod. 1985, 48, 429.

33. Sasamori, H.; Heddy, K. S.; Kirkkup, M. P.; Shabanowitz, J.; Lynn, D. V.; Hecht, S. M.; Woode, K. A.; Bryan, R. F.; Campbell, J.; Lynn, W. S.; Egert, E.; Sheldrick, G. M.; J. Chem. Soc., Perkin Trans. 1 1983, 1333.

34. Budzikiewiez, H.; Djerassi, C.; Williams, D. H.; Structure Elucidation of Natural Products by Mass Spectrometry, Holden-Day: San Francisco, 1964, vol. 2 , cap. 23

35. Audier, H. E.; Das, B. C.; Tetrahedron Lett. 1966, 2205.

36. Kupchan, S. M.; Smith, R. M.; Aynehchi, Y.; Maruyama, M.; J. Org. Chem. 1970, 35, 2891.

37. Johnson, L. B.; Reese, P, B.; Roberts, E. V.; Lam, L. K. P.; Vederas, J. C.; J. Chem. Soc., Perkin Trans. 1 1989, 2111.

38. Johnson, L. B.; Griffiths, W. J.; Roberts, E. V.; Lam, L. K. P.; Vederas, J. C.; Reid, C. J.; Ballantine, J. A.; J. Chem. Soc., Perkin Trans. 1 1991, 2583.

39. Okabe, H.; Miyahara, Y.; Yamauchi, T.; Miyahara, K.; Kawasaki, T.; Chem. Pharm. Bull. 1980, 28, 2753.

40. Nes, W. D.; Wong, R. Y.; Benson, M.; Akihisa, T.; J. Chem. Soc., Chem. Commun. 1991, 1272

41. Sturm, S.; Stuppner, H.; Phytochem. Anal. 2000, 11, 121.

42. Miyahara, Y.; Okabe, H.; Yamauchi, T.; Chem. Pharm. Bull. 1981, 29, 1561.

43. Hylands, P. J.; Kosugi, J.; Phytochemistry 1982, 21, 1379.

44. Oobayashi, K.; Yoshikawa, K.; Arihara, S.; Phytochemistry 1992, 31, 943.

45. Kasai, R.; Matsumoto, K.; Nie, R-L.; Morita, T.; Awazu, A.; Zhou, J.; Tanaka, O.; Phytochemistry 1987, 26, 1371.

46. Fatope, M. O.; Takeda, Y.; Yamashita, H.; Okabe, H.; Yamauchi, T.; J. Nat. Prod. 1990, 53, 1491.

47. Akihisa, T.; Yasukawa, K.; Kimura, Y.; Takido, M.; Kokke, W. C. M. C.; Tamura, T.; Phytochemistry 1994, 36, 153.

48. Mulholland, D. A.; Sewram, V.; Osborne, R.; Pegel, K. H.; Connolly, J. D.; Phytochemistry 1997, 45, 391.

49. Achenbach, H.; Horn, K.; Dominguez, X. A.; Rombold, C.; Lopez, E. G. G.; Phytochemistry 1993, 33, 437.

50. Huang, Y.; Bruyne, T. D.; Apers, S.; Ma, Y.; Clayes, M.; Berghe, D. V.; Pieters, L.; Vlietinck, A.; J. Nat. Prod. 1998, 61, 757. 
51. Mai, L. P.; Guénard, M.; Franck, M.; Tri, M. V.; Gaspard, C.; Sévenet, T.; Nat. Prod. Lett. 2002, 16, 15.

52. Stuppner, H.; Müller, E. P.; Phytochemistry 1993, 33, 1139

53. Gamlath, C. B.; Gunatilaka, L. A. A.; Alvi, K. A.; Atta-ur-Rahman; Sinnathamby, B.; Phytochemistry 1988, 27, 3225.

54. Hylands, P. J.; Mansour, E-S. S.; Phytochemistry 1982, 21, 2703.

55. Stuppner, H.; Kählig, H.; Seligmann, O.; Wagner, H.; Phytochemistry 1990, 29, 1633.

56. Yamada, Y.; Hagiwara, K.; Iguchi, K.; Tetrahedron Lett. 1977, 2099.

57. Kupchan, S. M.; Sigel, C. W.; Guttmann, L. J.; Restivo, R. J.; Bryan, R. F.; J. Am. Chem. Soc. 1972, 94, 1353.

58. Bittner, M.; Poyser, A.; Poyser, J. P.; Silva, M.; Weldt, E.; Sammes, P. G.; Phytochemistry 1973, 12, 1427.
59. Hylands, P. J.; Magd, M. S.; Phytochemistry 1986, 25, 1681.

60. Reddy, K. S.; Amonkar, A. J.; McCloud, T. G.; Chang, C-J.; Cassady, J. M.; Phytochemistry 1988, 27, 3781 .

61. Sachdev-Gupta, K.; Radke, C. D.; Renwick, A. A.; Phytochemistry 1993 33, 1385.

62. Kupchan, S. M.; Meshulam, H.; Snedem, A. T.; Phytochemistry 1978, 17, 767.

63. Laurie, W. A.; McHale, D.; Sheridan, J. B.; Phytochemistry 1985, 24, 2659

64. El-Fattah, H. A.; Phytochemistry 1994, 36, 159.

65. Rodriguez, N.; Vasquez, Y.; Hussein, A. A.; Colley, P. D.; Solis, P. N.; Gupta, M. P.; J. Nat. Prod. 2003, 66, 1515. 